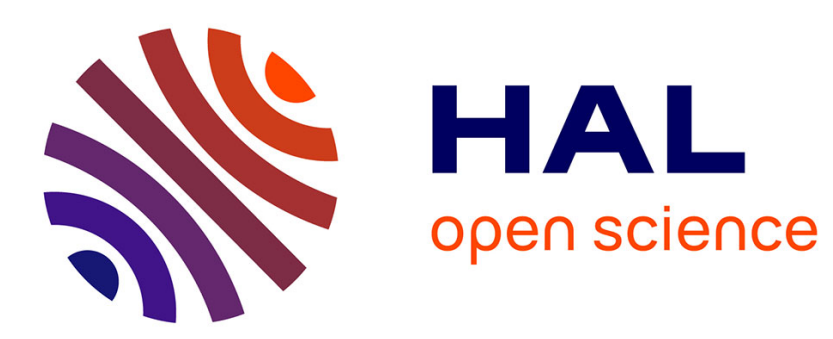

\title{
Side-chain liquid-crystalline polyacrylates: Experimental evidence of a coexistence of a double main-chain confinement inside the smectic layer
}

Laurence Noirez

\section{- To cite this version: \\ Laurence Noirez. Side-chain liquid-crystalline polyacrylates: Experimental evidence of a coexistence of a double main-chain confinement inside the smectic layer. EPL - Europhysics Letters, 1999, 46 (6), pp.728-734. hal-01361919}

\author{
HAL Id: hal-01361919 \\ https://hal.science/hal-01361919
}

Submitted on 8 Sep 2016

HAL is a multi-disciplinary open access archive for the deposit and dissemination of scientific research documents, whether they are published or not. The documents may come from teaching and research institutions in France or abroad, or from public or private research centers.
L'archive ouverte pluridisciplinaire HAL, est destinée au dépôt et à la diffusion de documents scientifiques de niveau recherche, publiés ou non, émanant des établissements d'enseignement et de recherche français ou étrangers, des laboratoires publics ou privés. 
Side-chain liquid-crystalline polyacrylates: Experimental evidence of a coexistence of a double main-chain confinement inside the smectic layer

This content has been downloaded from IOPscience. Please scroll down to see the full text. 1999 Europhys. Lett. 46728

(http://iopscience.iop.org/0295-5075/46/6/728)

View the table of contents for this issue, or go to the journal homepage for more

Download details:

IP Address: 132.166.24.22

This content was downloaded on 24/05/2016 at $13: 13$

Please note that terms and conditions apply. 


\title{
Side-chain liquid-crystalline polyacrylates: Experimental evidence of a coexistence of a double main-chain confinement inside the smectic layer
}

\author{
L. NOIREZ \\ Laboratoire Léon Brillouin (CEA-CNRS), Ce-Saclay \\ 91191 Gif-sur-Yvette, Cédex, France \\ (received 24 August 1998; accepted in final form 19 April 1999) \\ PACS. 36.20Ey - Conformation (statistics and dynamics). \\ PACS. 61.12Ex - Neutron scattering techniques. \\ PACS. 61.12Ld - Single-crystal and powder diffraction.
}

\begin{abstract}
Detailed profiles providing the main-chain/mesogen organisation within the smectic layers have been deduced from neutron diffraction measurements, by combination of the coherent scattering length profiles of the fully hydrogenated and the partially deuterated sidechain liquid-crystal polymers. Two different sites of the molecule (either on the main chain or on the mesogen extremity) have been labelled to prove that main chains can occupy the central zone of the mesogenic layers whereas most of them remains confined between the layers of mesogens. This surprising result can be explained considering symmetry arguments.
\end{abstract}

Introduction. - Side-chain liquid crystalline polymers are made of lateral liquid crystalline graftings fixed onto a main chain via a spacer. This structure allows the formation of a broad variety of liquid crystalline phases; the simplest ones are the nematic and smectic phases. This last one designates the establishment of defined long-range layers containing, for example, rod-like mesogens gathered parallel to each other ( $S_{\mathrm{A}}$ phase).

In the case of side-chain liquid-crystalline polymers, the smectic phase is a result of the packing of the side-chain land so the primary question in such systems is to know where and how the main chains are arranged. The picture usually accepted is that the main chains and the side chains remain, on average, perpendicular to each other, thus repelling the main chains from the mesogenic zone and concentrating them at the interfaces. This intuitive picture was adopted as a basic assumption for the theoretical predictions concerning the main-chain anisotropy in the smectic phase [1]. The first observations of the main-chain conformation (carried out by Small-Angle Scattering [2]) shows that the main-chain extension is favoured in the direction perpendicular to the director which is in good agreement with the predictions.

The first determinations of side-chain liquid-crystalline profiles were carried out by X-ray scattering giving rise to electronic density profiles [3]. These profiles effectively indicate an increase of electronic density on both sides of the mesogenic layer which was interpreted as being due to the high concentration of main chains. An additional increase in the middle of the layers has also been noticed by X-ray techniques, but was interpretated as being due to the high electronic density of the phenyl rings of the mesogen. Assumptions are therefore needed 


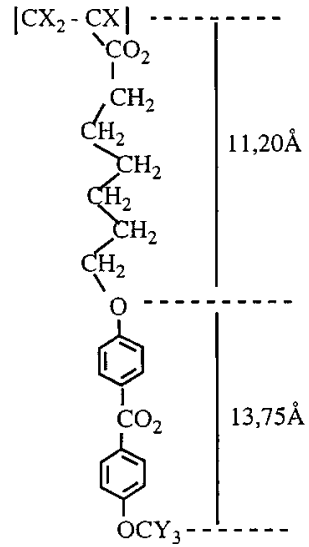

Fig. 1

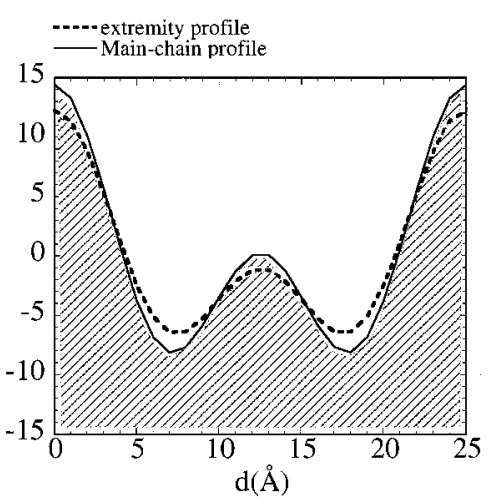

Fig. 2

Fig. 1. - Chemical formula of the liquid-crystalline polyacrylate. The distances associated to the spacer and the hard core are specified.

Fig. 2. - Profile of distribution of the main chains (continuous line) and of the mesogen extremities$\mathrm{OCD}_{3}$ (dashed line) along the $\mathrm{Oz}$ axis of one smectic layer (thickness $25 \AA$ ) at room temperature.

to deduce the localisation of the electronic density associated to the main chains $[3,4]$. In contrast, the use of neutron scattering with a specific labelling allows the determination of the exact main-chain localisation in the smectic layer. This method was first successfully employed on a powder of a liquid crystal polymer at room temperature [1], and later a complete study on an aligned sample of liquid-crystalline polymer has been carried out vs. temperature [5].

A rapid overview of the results obtained by these methods shows that a rich variety of smectic profiles can be obtained with liquid-crystalline polymers often only slightly different. The common denominator remains however the high concentration of polymer main chains between the mesogen layers. It is also reported that minor changes in the polymer structure (addition or loss of the methyl group along the main chain, variation of the extremity length...) strongly affect the distribution profiles of the main chains [6]. The experimental description reveals a much more complex organisation than the one proposed by the theoretical models.

This paper illustrates how far the experimental profiles can be from the basic picture of thin main-chains layers forming the interface between two well-defined mesogenic layers. In particular, it will be demonstrated that the main chains can even be concentrated in the central zone of the mesogen layers. We will focus on the behaviour in the smectic phase of a liquid-crystalline polyacrylate which has developed this ability. This description is based on the determination by neutron diffraction of the main-chain distribution profile obtained at different temperatures. The polyacrylate described here was previously studied by smallangle neutron scattering providing the average main-chain dimensions following the directions parallel and perpendicular to the director [7]. This study confirmed that in the smectic phase, the strong anisotropy of the main chain $\left(R_{\|}<R_{\perp}\right)$ expresses the main-chain propensity to be perpendicular to the mesogen orientation.

Experimental. - The polymer is the same as the one described in [7]. It has been synthesized by radical polymerization [8] and corresponds to the structural formula illustrated in fig. 1 where the sites $\mathrm{X}, \mathrm{Y}=\mathrm{H}$ or D are either hydrogenated or deuterated. Three types of labelling have been used:

1) the fully hydrogenated polymer (I): $\mathrm{X}=\mathrm{Y}=\mathrm{H}$ noted $\mathrm{PA}(\mathrm{H})-\mathrm{OCH}_{3}$ with $M_{\mathrm{w}}=26800$, $I=2.6$; 
2) the polymer deuterated on the main chain (II): $\mathrm{X}=\mathrm{D}, \mathrm{Y}=\mathrm{H}$ noted $\mathrm{PA}(\mathrm{D})-\mathrm{OCH}_{3}$ with $M_{\mathrm{w}}=41000, I=2$;

3 ) the polymer deuterated at the end of the mesogen (III): $\mathrm{X}=\mathrm{H}, \mathrm{Y}=\mathrm{D}$ noted $\mathrm{PA}(\mathrm{H})-\mathrm{OCD}_{3}$ with $M_{\mathrm{w}}=27250, I=2.2$.

The three isotopic variants display the same phase diagram (within $\pm 2{ }^{\circ} \mathrm{C}$ ): $T_{\mathrm{g}}$ (glassy) $35{ }^{\circ} \mathrm{C}-\mathrm{S}_{\mathrm{A} 1}$ (smectic)- $89{ }^{\circ} \mathrm{C}-\mathrm{N}$ (nematic)- $116{ }^{\circ} \mathrm{C}-\mathrm{I}$ (isotropic) (measured by DSC with a temperature rate of $\left.-5{ }^{\circ} \mathrm{C} / \mathrm{min}\right)$.

Three disc-like samples (15 $\mathrm{mm}$ diameter, $1 \mathrm{~mm}$ thickness) were prepared containing, respectively, the fully hydrogenated polymer (I), the polymer deuterated on the mesogen extremity (III) and a 1:1 mixture of fully hydrogenated polymer and of polymer deuterated on the main chain alone (I+ II).

The neutron diffraction measurements were achieved by slowly decreasing the temperature from the isotropic phase down to the glassy state under a magnetic field of $1.4 \mathrm{~T}$. The same alignment process was applied for each sample.

The neutron scattering equipment used for the experiment was the 2-dimensional multidetector PAXY of the Léon Brillouin Laboratory adjusted in order to give access to the scattering range: $0.02 \AA^{-1}<|\vec{q}|<0.5 \AA^{-1}$ (multidetector-sample distance: $1 \mathrm{~m}$, wavelength: $5 \AA$ ). The sample aligned by a horizontal magnetic field, was rotated around its vertical axis in order to determine the maximum intensity of the smectic reflections (corresponding to the Bragg conditions). This position was determined at low temperature, by the measurement of the variation of the smectic reflections vs. the rotation angle (rocking curve).

Data exploitation: basic assumptions for the profile determination. - The profile construction is worked out from a $2 \mathrm{~d}$ determination of the intensity integrated on zone including each smectic reflection measured at Bragg conditions. The background noise (incoherent scattering, electronic noise...) is subtracted by measuring the intensity received on the multidetector in a background zone equivalent to the one defined for the smectic reflections. All these measurements have been carried out on corrected 2-dimensional data of the multidetector (previously normalised by an incoherent scatterer and compared to the same number of scatterers).

The following table gives the results obtained on aligned samples using this procedure:

TABLE I. -

\begin{tabular}{lcc}
\hline $\begin{array}{l}\text { Smectic reflection intensities - } \\
\text { amplitude (room temperature): }\end{array}$ & $\begin{array}{c}\text { 001 intensity (a.u.) } \pm 5 \% \\
\mid \text { Amplitude } \mid\end{array}$ & $\begin{array}{c}\text { 002 intensity (a.u.) } \pm 5 \% \\
\mid \text { Amplitude } \mid\end{array}$ \\
\hline $\mathrm{PA}(\mathrm{H})-\mathrm{OCH}_{3}$ & 25.55 & 3.55 \\
& $|\mathrm{~A} 001|=5.09$ & $|\mathrm{~A} 002|=1.90$ \\
\hline $\mathrm{PA}(\mathrm{H})-\mathrm{OCD}_{3}$ & 3.49 & 13.33 \\
& $|\mathrm{~A} 001|=1.57$ & $|\mathrm{~A} 002|=3.67$ \\
\hline $\mathrm{PA}(\mathrm{H}) / \mathrm{PA}(\mathrm{D})-\mathrm{OCH}_{3}$ & 1 & 7.22 \\
$1: 1$ & $|\mathrm{~A} 001|=1$ & $|\mathrm{~A} 002|=2.71$ \\
\hline
\end{tabular}

The intensity of the 001 reflection of the H/D mixture has been determined using a twodimensional fit to take into account together the form factor of the main chain (central scattering) and the smectic reflections [9].

Whatever the labelling type or the scattering technique (neutron or X-ray [10]), only two smectic reflections have been detected. The absence of higher orders is then not due to an extinction in the structure factor. 
The procedure adopted to determine the distribution profile of the main chains in the smectic layers is based on the following assumptions. The smectic A phase is supposed to be a continuous periodic medium without a preferred mesogen orientation (up or down), it can then be described by a Fourier series expanded with cosine terms:

$$
\rho(z)=\rho_{0}+\sum_{i} A_{i} \cos \left(\frac{2 \pi}{d} i\right)\left(\mathrm{cm}^{-1}\right),
$$

where $d$, the layer thickness, is $25 \AA$ in the case of this polymer. $\rho_{0}$ is the average density of coherent scattering and $\rho(z)$ is the variation of the density along the director $O z$.

The scattering function associated to this modulation is obtained via the Fourier transform. The intensity of the smectic reflections is then assimilated to the square of the term of amplitude $\left|A_{n}\right|$. The normalized values of $\left|A_{n}\right|$, measured at room temperature, are listed in table $\mathrm{I}$.

The determination of the profile of coherent scattering length then consists of the reconstruction of the Fourier series using the $\left|A_{n}\right|$ values and determining each associated sign. Every possible combination of sign is examined and only the ones which have a physical meaning are kept. In order to find more easily the right sign combination, each profile obtained for a given combination is compared to a theoretical profile [6], calculated on the basis of the scattering length density produced by each element: main chain, spacer and hard core.

\section{Results - discussion}

Determination of the main-chain profile. - The profile of the main-chain distribution $\Delta \rho_{\text {main chain }}(z)$, is deduced from the subtraction of the function corresponding to the $\mathrm{H} / \mathrm{D}$ mixture from the function corresponding to the fully hydrogenated polymer (each density has been normalised to the same number of scatterers) $\Delta \rho_{\text {main chain }}(z)=2 \rho_{\mathrm{H} / \mathrm{D}}(z)-$ $\rho_{\mathrm{H}}(z)-\rho_{0}$, where

$$
\begin{aligned}
\rho_{\mathrm{H} / \mathrm{D}}(z) & =\rho_{0}+\cos \left(\frac{2 \pi}{25} z\right)+2.71 \cos \left(\frac{4 \pi}{25} z\right), \\
\rho_{\mathrm{H}}(z) & =\rho_{0}-5.09 \cos \left(\frac{2 \pi}{25} z\right)-1.90 \cos \left(\frac{4 \pi}{25} z\right) .
\end{aligned}
$$

The resulting function is presented in fig. 2 (continuous line) and corresponds to the density of presence of main chains along the director $O z$ within one mesogenic layer $(25 \AA)$. The excess density on each side of the diagram corresponds to the main-chain confinement between the mesogen layers. The profile shows in addition a small bump in the middle of the mesogenic layer which has to be associated to an increase of main-chain density in the central zone. The presence of main chains in this central part is directly related to the exceptionally strong intensity of the 002 reflection. The 002 reflection is exacerbated in the case of the H/D mixture but not for the fully hydrogenated polymer, or by X-ray scattering. The specificity of the neutron interaction with isotopic nuclei is particularly well adapted to reveal the internal composition of the smectic layers since the profile combination avoids the question of structure factor extinction.

Determination of the distribution profile of mesogen extremities. The study of the polymer deuterated on the extremity of the mesogen gives rise, by combination with the profile obtained for the fully protonated sample, to the profile of distribution of the extremities in the smectic layer. The localisation of main chains between and in the central part of the mesogen layers implies reciprocally (and since the mesogens are in their most 


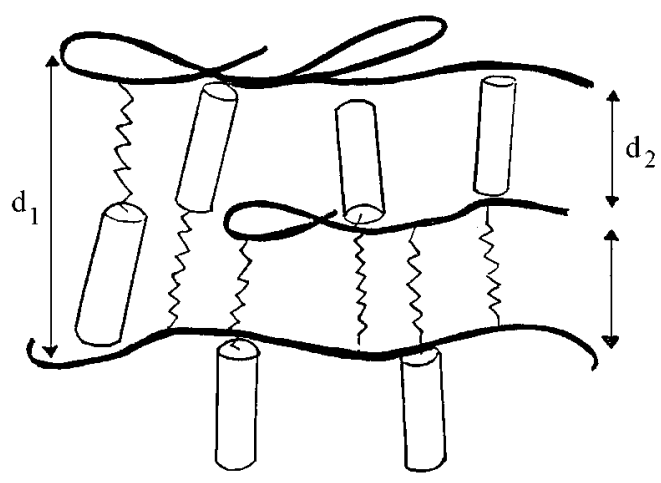

Fig. 3. - Schematic representation of the organization in "double-layer" packing of the liquidcrystalline polyacrylate.

elongated configuration) that the mesogen extremities have to be also concentrated in the central part of the layers and between them. The only physical solution acceptable to describe the density distribution associated to the polymer $\mathrm{PA}(\mathrm{H})-\mathrm{OCD}_{3}$ is, using the data of table I:

$$
\rho_{\mathrm{OCD}_{3}}(z)=\rho_{0}+1.57 \cos \left(\frac{2 \pi}{25} z\right)+3.67 \cos \left(\frac{4 \pi}{25} z\right) .
$$

The second order is obviously the predominating one. The profile associated to the distribution of mesogen extremities is obtained by subtracting the fully hydrogenated polymer profile. It corresponds to the following function illustrated in fig. 2 by the dashed line:

$$
\Delta \rho_{\text {ext.D }}(z)=\rho_{\mathrm{OCD}_{3}}(z)-\rho_{\mathrm{H}}(z)=6.66 \cos \left(\frac{2 \pi}{25} z\right)+5.57 \cos \left(\frac{4 \pi}{25} z\right) .
$$

This figure is very similar to the main-chain distribution. The profile also indicates the presence of higher concentration of extremities (and hence of main chains) on both sides of the mesogenic layer but also in the central part which confirms the double layer packing.

Interpretation. - The results obtained from two independent studies (main-chain distribution or extremity distribution) give rise to the same conclusion; the presence of a certain amount of polymer main chains in the central part of the mesogen layer. Does this sound from a physical point of view? First, one can exclude artefact effects such as multiple scattering which could exacerbate the 002 reflection, since at high temperature the hydrogenated polymer displays only one strong 001 smectic reflection. From a CKP molecular model, it can be seen that the spacer and the hard core correspond almost to the same lengths (fig. 1). As indicated in fig. 3, a subdivision of the layer is allowed by the symmetry since both lengths are about $12 \AA$. Moreover, this molecular structure favours the interactions between alkyl parts (and reciprocally between hard cores). The sublayer arrangement reduces the repetitive unit to half of its previous value by introducing a new symmetry in the layer and then enhancing the 002 smectic reflection.

This picture is supported by another structural detail which comes from the analysis of the shape presented by the smectic peaks of the polymer deuterated at the extremity $\left(\mathrm{PA}^{-} \mathrm{OCD}_{3}\right)$. The scattering observed along the meridian for this polymer at low temperature shows a splitting in the smectic reflections; the 002 and the 001 smectic reflections contain actually the superposition of two peaks which appear in fig. 4a) as shoulders of the main peaks. Different conjugations of wavelengths and distances have been tested to improve the definition $(\lambda=7 \AA$, 

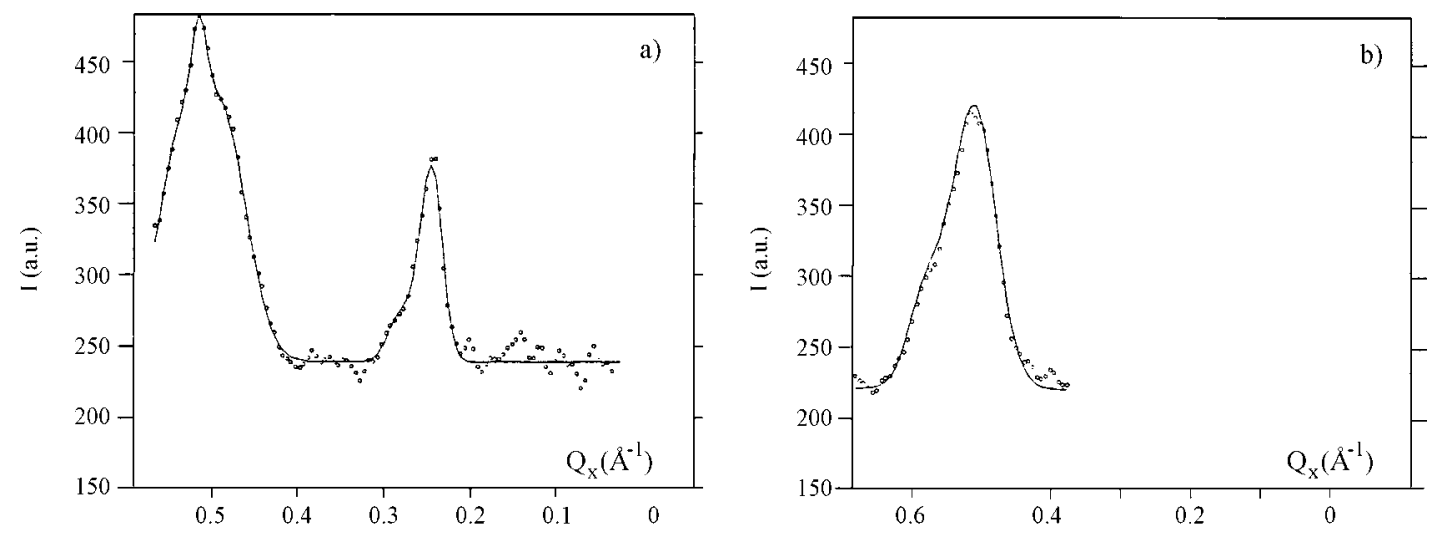

Fig. 4. - Side view of the scattering observed along the meridian for the $100 \% \mathrm{D}$ PA-OCD 3 . a) At $50{ }^{\circ} \mathrm{C}$, after the alignment process $(\lambda=5 \AA, d=1.25 \mathrm{~m})$. b) Room temperature, some days later $(\lambda=5 \AA, d=1 \mathrm{~m})$.

$d=2 \mathrm{~m}$ ) and ascertain the existence of two contributions. Moreover, the relative intensity of these two maxima evolves with time at room temperature, resulting in a further increase of the one at smaller $q$-values (fig. 4b)). These two maxima are, respectively, at $|\vec{q}|=0.531 \AA^{-1}$ and at $|\vec{q}|=0.510 \AA^{-1}$. The last value $\left(|\vec{q}|=0.510 \AA^{-1}\right)$ corresponds to a distance of $12.33 \AA$ which can be identified as the 002 reflection (from 3-axis spectrometer experiments, the layer distance measured on the fully hydrogenated polymer is about $24.5 \AA \pm 0.01 \AA$ ). The same observation can be carried out on the 001 reflection (centred at $|\vec{q}|=0.255 \AA^{-1}$ ) which displays a shoulder at large $q$-values $\left(|\vec{q}|=0.287 \AA^{-1}\right)$.

These satellites around the fundamental orders reveal the coexistence of different characteristic lengths following the meridian. It could present some similarities with systems called incommensurate smectics. The structure presented by the mesogens attached to both sides of a main chain is reminiscent of the dimesogen architecture [11]. However, it seems to be hazardous to state the existence of superposed structures, since these two distinct modulations appear only by neutron scattering and in the case of the polymer deuterated on the extremity but neither on the fully hydrogenated one, nor by X-ray scattering.

The specific labelling makes new contrasts appear between the hydrogenated and the deuterated sites within the layer. In particular, one can define two specific distances which are frequently repeated. They correspond to the distance, along the director, separating the nearest labelled parts which is effectively slightly smaller than the layer thickness and one half of it for the sublayer, respectively (fig. 3). These distances fit well with the experimental values found for the additional scattering (11.81 $\AA$ and $21.88 \AA$ ). This means that the scattering observed for the polymer deuterated on the extremities contains two components of distinct origin, which are the long-range smectic correlation yielding the usual Bragg reflection, and an additional pair correlation corresponding to the sum of local interactions between the labelled parts within each layer. The question wether it is still correct to integrate this extra-scattering into the calculation of the profiles is unclear. However, even if it should be taken into account, the origin of this scattering is a supplementary strong indication in favour of the double layer packing.

Conclusions. - We claim that main chains cannot only be confined to both sides of mesogenic layers but also centred in a defined zone corresponding to the median part of the mesogen layer. 
The demonstration is based on the determination of coherent scattering length profiles specifically associated either to the main-chain distribution or to the mesogen extremity distribution along the director $O z$. In both cases (which can be considered as two independent procedures), the result is an increase of main-chain concentration between the mesogen layer (simple layer packing and simple main-chain confinement) and a weaker increase of main-chain concentration extended over $8 \AA$ in the central part of the mesogen layer (sublayer packing and double main-chain confinement). The sublayer packing is allowed by the symmetry between the spacer and the hard core which optimises the interactions between segments of the same species. This is the first time that such an arrangement is clearly established. This result is probably related to the high flexibility of the main-chain and perhaps also to the chain length since the polymethacrylate homologues (possessing an identical side-chain mesogen) corresponds to a single sine wave, which is the less organised smectic structure [6]. Other studies have also shown the existence of peculiar transitions in the smectic phase owing to the presence of the polymer [12] but no double main-chain confinement has yet been observed up to now.

Finally, a detailed analysis of the shape of the smectic reflections of the polymer deuterated on the extremity shows a peak splitting. The additional intensity is interpreted by the contribution of the local but well-defined interactions between labelled centres. This confirms the existence of the sublayer arrangements previously deduced. The use of a spectrometer (3axis) specially adapted to accurate elastic measurements is needed to provide more information on the exact peak shapes, the real correlation lengths associated to each of them and the corresponding transition temperatures.

To our knowledge, the $\mathrm{PA}-\mathrm{OCH}_{3}$ is the only polymer which displays this particularity. It would be of interest to know if this behaviour extends to other liquid-crystalline polymers.

The author would like to thank P. KELLER for providing the hydrogenated and the deuterated polymers, and F. Moussa and M. QuiLICHINI for stimulating discussions.

\section{REFERENCES}

[1] a) Renz W. and Warner M., Phys. Rev. Lett., 56 (1986) 1268; b) Rieger J., Mol. Cryst. Liquid Cryst., 155 (1988) 253; c) Rieger J., J. Phys. (Paris), 49 (1988) 253.

[2] a) Kirste R. G. and Ohm H. G., Makromol. Chem. Rapid Commun., 6 (1985) 179; b) Keller P., Carvalho B., Cotton J. P., Lambert M., Moussa F. and Pépy G., J. Phys. Lett., 46 (1985) 1065.

[3] Gudkov V. A., Sov. Phys. Crystallogr., 29 (1984) 316.

[4] a) Davidson P. and Strzelecki L., Liquid Cryst., 2 (1988) 1583; b) Davidson P., Keller P. and Levelut A. M., J. Phys. (Paris), 46 (1985) 939; c) Davidson P., Levelut A. M., Achard M. F. and Hardouin F., Liquid Cryst., 4 (1989) 561.

[5] Noirez L., Davidson P., Schwarz W. and PÉPy G., Liquid Cryst., 16 (1994) 1081.

[6] Noirez L., Mol. Cryst. Liquid Cryst., 261 (1995) 525.

[7] Noirez L., Cotton J. P., Hardouin F., Keller P., Moussa F., Pépy G. and Strazielle C., Macromolecules, 21 (1988) 1383.

[8] a) Portugall M., Ringsdorf H. and Zentel R., Makromol. Chem., 183 (1982) 2311; b) Zugenmaier Mugge P., Makromol. Chem. Rapid Commun., 5 (1984) 11.

[9] PÉPY G, Program "PXY" available at the LBB.

[10] Davidson P. and Levelut A. M., Liquid Cryst., 11 (1992) 469.

[11] Attard G. S., Date R. W., Imrie C. T., Luckhurst G. R., Roskilly S., Sedden J. M. and TAYLOR L., Liquid Cryst., 16 (1994) 529.

[12] Tahanari Yamagushi T. and Asada T., Liquid Cryst., 10 (1991) 215. 